\title{
Universiteit
}

The Netherlands

\section{Relation between entanglement measures and Bell inequalities for three qubits}

Emary, C.; Beenakker, C.W.J.

\section{Citation}

Emary, C., \& Beenakker, C. W. J. (2004). Relation between entanglement measures and Bell

inequalities for three qubits. Physical Review A, 69, 032317. doi:10.1103/PhysRevA.69.032317

Version: $\quad$ Not Applicable (or Unknown)

License: $\quad$ Leiden University Non-exclusive license

Downloaded from: https://hdl.handle.net/1887/61257

Note: To cite this publication please use the final published version (if applicable). 


\title{
Relation between entanglement measures and Bell inequalities for three qubits
}

\author{
C. Emary and C. W. J. Beenakker \\ Instituut-Lorentz, Universiteit Leiden, P.O. Box 9506, 2300 RA Leiden, The Netherlands
}

(Received 17 November 2003; published 23 March 2004)

\begin{abstract}
For two qubits in a pure state there exists a one-to-one relation between the entanglement measure (the concurrence $\mathcal{C}$ ) and the maximal violation $\mathcal{M}$ of a Bell inequality. No such relation exists for the three-qubit analog of $\mathcal{C}$ (the tangle $\tau$ ), but we have found that numerical data is consistent with a simple set of upper and lower bounds for $\tau$ given $\mathcal{M}$. The bounds on $\tau$ become tighter with increasing $\mathcal{M}$, so they are of practical use. The Svetlichny form of the Bell inequality gives tighter bounds than the Mermin form. We show that the bounds can be tightened further if the tangle is replaced by an entanglement monotone that can identify both the $\mathrm{W}$ state and the Greenberger-Horne-Zeilinger state.
\end{abstract}

DOI: $10.1103 /$ PhysRevA.69.032317

PACS number(s): 03.67.Mn, 03.65.Ud

Bell inequalities test for the quantum entanglement of a state by comparing the maximally measured value $\mathcal{M}$ of a certain correlator with the maximal value allowed by local realism [1]. For a pure state of two qubits, the Bell-CHSH (Clauser-Horne-Shimony-Holt [2]) parameter $\mathcal{M}=2 \sqrt{1+\mathcal{C}^{2}}$ is directly related to the degree of entanglement (or concurrence) $\mathcal{C} \in[0,1]$ of the state [3]. This relation is useful because, on the one hand, $\mathcal{M}$ can be readily measured [4], while on the other, $\mathcal{C}$ can be readily calculated [5]. In this paper we investigate to what extent this relation has a threequbit analog.

The three-qubit analog of the concurrence $\mathcal{C}$ is the tangle $\tau$, introduced by Coffman, Kundu, and Wootters [6]. It quantifies the irreducible tripartite entanglement through the formula

$$
\tau=\mathcal{C}_{A(B C)}^{2}-\mathcal{C}_{A B}^{2}-\mathcal{C}_{A C}^{2}
$$

The indices $A, B, C$ label the three qubits; the tangle is invariant under permutation of these indices. The concurrence $\mathcal{C}_{A B}$ refers to the mixed state of qubits $A$ and $B$ obtained after tracing out the degree of freedom of qubit $C$, and $\mathcal{C}_{A C}$ is defined similarly. The concurrence $\mathcal{C}_{A(B C)}$ describes the entanglement of qubit $A$ with the joint state of qubits $B$ and $C$. The tangle $\tau \in[0,1]$ equals 0 if one of the qubits is separable from the other two. It equals 1 for the maximally entangled GHZ (Greenberger-Horne-Zeilinger [7]) state $|\psi\rangle_{\mathrm{GHZ}}$ $=(|000\rangle+|111\rangle) / \sqrt{2}$.

The best-studied generalization of the Bell-CHSH inequality to the case of three qubits is the one proposed by Mermin [8]. There exists no analytical formula that gives the maximal violation $\mathcal{M}_{\mathrm{M}}$ of the Mermin inequality for a given pure state of three qubits, but it is not difficult to perform the maximization numerically. For special one-parameter states of the form $|\psi\rangle=\cos \alpha|000\rangle+\sin \alpha|111\rangle$, Scarani and Gisin [9]found an approximate (but highly accurate) relation $\mathcal{M}_{\mathrm{M}} \approx \max (4 \sqrt{\tau, 2 \sqrt{1-\tau}})$ between $\tau=\sin ^{2} 2 \alpha$ and $\mathcal{M}_{\mathrm{M}}$.

For more general states there is a range of values of $\tau$ with the same $\mathcal{M}_{\mathrm{M}}$. We have investigated this range numerically and found that the data is well described by a simple pair of upper and lower bounds for $\tau$ for any given $\mathcal{M}_{\mathrm{M}}$. The bounds can be tightened in two ways: (1) By using an alter- native form of the three-qubit Bell inequality, due to Svetlichny [10-13]; and (2) by using an alternative measure $\sigma$ of tripartite entanglement that we introduce in this paper, defined by

$$
\sigma=\min \left(\frac{\mathcal{C}_{X(Y Z)}^{2}+\mathcal{C}_{Y(X Z)}^{2}}{2}-\mathcal{C}_{X Y}^{2}\right)
$$

The minimization is over the permutations $X, Y, Z$ of the qubits $A, B, C$. We find the following bounds on $\sigma$ for a given maximal violation $\mathcal{M}_{\mathrm{S}}$ of the Svetlichny inequality:

$$
\left|\mathcal{M}_{\mathrm{S}}^{2} / 16-1\right| \lesssim \sigma \lesssim \mathcal{M}_{\mathrm{S}}^{2} / 32 \text {. }
$$

(We use the symbol $\lesssim$ instead of $\leqslant$ as these bounds are inferred from numerical data, rather than derived analytically.)

Both $\sigma$ and $\tau$ are entanglement monotones (meaning that they cannot be increased on average by local operations and classical communication). Their essential difference is that $\sigma$ can detect tripartite entanglement of both the W and GHZ types, while it is known that $\tau$ can only detect GHZ type entanglement [14]. We recall that local operations on the $\mathrm{W}$ state $|\psi\rangle_{\mathrm{W}}=(|001\rangle+|010\rangle+|100\rangle) / \sqrt{3}$ and the GHZ state $|\psi\rangle_{\mathrm{GHZ}}$ generate two distinct classes of irreducibly entangled tripartite states. While $\tau=1=\sigma$ for $|\psi\rangle_{\mathrm{GHZ}}$, for $|\psi\rangle_{\mathrm{W}}$ only $\sigma$ $=4 / 9$ is nonzero. In fact, $\sigma=0$ if and only if one of the qubits is separable from the other two (2-1 separability). This latter property distinguishes the entanglement measure introduced here from the one introduced by Meyer and Wallach [15], which is also nonzero for 2-1 separable states.

After this introduction, we now present our findings in more detail.

Pure states of three qubits constitute a five-parameter family, with equivalence up to local unitary transformations. This family has the representation [16]

$$
\begin{aligned}
|\psi\rangle= & \sqrt{\mu_{0}}|000\rangle+\sqrt{\mu_{1}} e^{i \phi}|100\rangle+\sqrt{\mu_{2}}|101\rangle+\sqrt{\mu_{3}}|110\rangle \\
& +\sqrt{\mu_{4}}|111\rangle,
\end{aligned}
$$

with $\mu_{i} \geqslant 0, \Sigma_{i} \mu_{i}=1$, and $0 \leqslant \phi \leqslant \pi$. The labels $A, B$, and $C$ indicate the first, second, and third qubit, while $X, Y, Z$ refer to an arbitrary permutation of these labels. 
The tangle (1) is given by

$$
\tau=4 \mu_{0} \mu_{4} .
$$

The squared concurrences $\mathcal{C}_{X(Y Z)}^{2}=4 \operatorname{Det} \rho_{X}$ (with $\rho_{X}$ $=\operatorname{Tr}_{Y, Z}|\psi\rangle\langle\psi|$ the reduced density matrix) take the form

$$
\begin{aligned}
& \mathcal{C}_{A(B C)}^{2}=4 \mu_{0}\left(\mu_{2}+\mu_{3}+\mu_{4}\right), \\
& \mathcal{C}_{B(A C)}^{2}=4 \mu_{0}\left(\mu_{3}+\mu_{4}\right)+4 \Delta, \\
& \mathcal{C}_{C(A B)}^{2}=4 \mu_{0}\left(\mu_{2}+\mu_{4}\right)+4 \Delta,
\end{aligned}
$$

with the definition $\Delta=\mu_{1} \mu_{4}+\mu_{2} \mu_{3}-2\left(\mu_{1} \mu_{2} \mu_{3} \mu_{4}\right)^{1 / 2} \cos \phi$. Each of the four quantities (5)-(8) is an entanglement monotone $[14,17]$.

The quantity $\sigma$ defined in Eq. (2) can equivalently be written as

$$
\sigma=\frac{1}{2}\left(\tau+\min \mathcal{C}_{Z(X Y)}^{2}\right)=\tau+\frac{1}{2} \min \left(\mathcal{C}_{X Z}^{2}+\mathcal{C}_{Y Z}^{2},,\right.
$$

as follows from the identity [18] $\tau=\mathcal{C}_{X(Y Z)}^{2}+\mathcal{C}_{Y(X Z)}^{2}-\mathcal{C}_{Z(X Y)}^{2}$ $-2 \mathcal{C}_{X Y}^{2}$. One sees that $0 \leqslant \tau \leqslant \sigma \leqslant 1$. Most importantly, since $\tau$ and $\min \mathcal{C}_{Z(X Y)}^{2}$ are positive entanglement monotones, their sum $\sigma$ is an entanglement monotone as well [19]. If one of the qubits $(Z)$ is separable from the other two, then $\tau=0=\mathcal{C}_{Z(X Y)} \Rightarrow \sigma=0$. The converse is also true: If $\sigma=0$ then $\mathcal{C}_{Z(X Y)}=0$ for some permutation $X, Y, Z$ of the qubits, so one qubit is separable from the other two.

Bell inequalities for three qubits are constructed from the correlator

$$
E(\boldsymbol{a}, \boldsymbol{b}, \boldsymbol{c})=\langle\psi|(\boldsymbol{a} \cdot \boldsymbol{\sigma}) \otimes(\boldsymbol{b} \cdot \boldsymbol{\sigma}) \otimes(\boldsymbol{c} \cdot \boldsymbol{\sigma})| \psi\rangle .
$$

Here $\boldsymbol{a}, \boldsymbol{b}, \boldsymbol{c}$ are real three-dimensional vectors of unit length that define a rotation of the Pauli matrices $\boldsymbol{\sigma}=\left(\sigma_{x}, \sigma_{y}, \sigma_{z}\right)$. One chooses a pair of vectors $\boldsymbol{a}, \boldsymbol{a}^{\prime}, \boldsymbol{b}, \boldsymbol{b}^{\prime}$, and $\boldsymbol{c}, \boldsymbol{c}^{\prime}$ for each qubit and takes the linear combinations

$$
\mathcal{E}=E\left(\boldsymbol{a}, \boldsymbol{b}, \boldsymbol{c}^{\prime}\right)+E\left(\boldsymbol{a}, \boldsymbol{b}^{\prime}, \boldsymbol{c}\right)+E\left(\boldsymbol{a}^{\prime}, \boldsymbol{b}, \boldsymbol{c}\right)-E\left(\boldsymbol{a}^{\prime}, \boldsymbol{b}^{\prime}, \boldsymbol{c}^{\prime}\right),
$$

$$
\mathcal{E}^{\prime}=E\left(\boldsymbol{a}^{\prime}, \boldsymbol{b}^{\prime}, \boldsymbol{c}\right)+E\left(\boldsymbol{a}^{\prime}, \boldsymbol{b}, \boldsymbol{c}^{\prime}\right)+E\left(\boldsymbol{a}, \boldsymbol{b}^{\prime}, \boldsymbol{c}^{\prime}\right)-E(\boldsymbol{a}, \boldsymbol{b}, \boldsymbol{c}) .
$$

Mermin's inequality [8] reads $|\mathcal{E}| \leqslant 2$, while Svetlichny's inequality $[10-13]$ is $\left|\mathcal{E}-\mathcal{E}^{\prime}\right| \leqslant 4$. We define the Mermin and Svetlichny parameters

$$
\mathcal{M}_{\mathrm{M}}=\max |\mathcal{E}|, \quad \mathcal{M}_{\mathrm{S}}=\max \left|\mathcal{E}-\mathcal{E}^{\prime}\right| .
$$

The maximization is over the six unit vectors $\boldsymbol{a}, \boldsymbol{b}, \boldsymbol{c}, \boldsymbol{a}^{\prime}, \boldsymbol{b}^{\prime}, \boldsymbol{c}^{\prime}$ for a given state $|\psi\rangle$. The largest possible value is reached for the GHZ state $\left(\mathcal{M}_{\mathrm{M}}=4\right.$ and $\left.\mathcal{M}_{\mathrm{S}}=4 \sqrt{2}\right)$. The $\mathrm{W}$ state has $\mathcal{M}_{\mathrm{M}} \cong 3.05$ and $\mathcal{M}_{\mathrm{S}} \cong 4.35$. Any violation of the Svetlichny inequality implies irreducible tripartite entanglement. In contrast, states in which one qubit is separable from the other two may still violate the Mermin inequality, up to $\mathcal{E}=2 \sqrt{2}$. For both inequalities, there exist pure entangled states that do not violate them $[9,20,21]$.

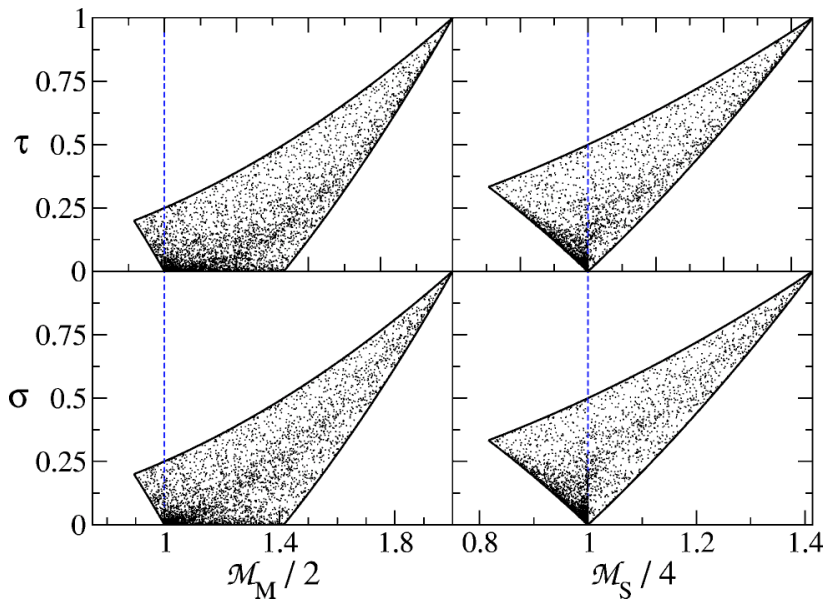

FIG. 1. Numerically determined Mermin $\left(\mathcal{M}_{\mathrm{M}}\right)$ and Svetlichny $\left(\mathcal{M}_{\mathrm{S}}\right)$ parameters for the three-parameter state (14). A range of values for the entanglement measures $\tau$ and $\sigma$ corresponds to the same value of $\mathcal{M}_{\mathrm{M}}$ or $\mathcal{M}_{\mathrm{S}}$. The solid curves are the upper and lower bounds (15) and (16). The dotted line indicates the maximum value obtainable with local variable theories.

The maximization over the two unit vectors $\boldsymbol{a}, \boldsymbol{a}^{\prime}$ can be done separately and analytically. The maximization over the remaining four unit vectors was done numerically. Before showing results for the full five-parameter family of states (4), it is instructive to first consider the three-parameter subfamily

$$
\begin{aligned}
|\Phi\rangle= & \cos \theta_{1}\left|\left(\begin{array}{l}
1 \\
0
\end{array}\right)\left(\begin{array}{l}
1 \\
0
\end{array}\right)\left(\begin{array}{l}
1 \\
0
\end{array}\right)\right\rangle \\
& +\sin \theta_{1}\left|\left(\begin{array}{l}
0 \\
1
\end{array}\right)\left(\begin{array}{c}
\cos \theta_{2} \\
\sin \theta_{2}
\end{array}\right)\left(\begin{array}{c}
\cos \theta_{3} \\
\sin \theta_{3}
\end{array}\right)\right\rangle,
\end{aligned}
$$

with real angles $\theta_{i}$. These states are all in the GHZ class, so for the moment we avoid the complication introduced by the W class. The physical significance of states of the form (14) is that they are generated in optical [22] or electronic [23] schemes to produce three-particle entanglement from two independent entangled pairs. (Notice that the second and third qubits become separable upon tracing over the first qubit.)

For any state of the form (14) picked at random, we calculate the two entanglement monotones $\tau$ and $\sigma$, and compute numerically the Mermin and Svetlichny parameters defined in Eq. (13). Results are plotted in Fig. 1. The numerical data fill a region bound by

$$
\begin{gathered}
\max \left(1-\frac{1}{4} \mathcal{M}_{\mathrm{M}}^{2}, 0, \frac{1}{8} \mathcal{M}_{\mathrm{M}}^{2}-1\right) \lesssim \tau, \sigma \lesssim \frac{1}{16} \mathcal{M}_{\mathrm{M}}^{2}, \\
\left|\frac{1}{16} \mathcal{M}_{\mathrm{S}}^{2}-1\right| \lesssim \tau, \sigma \lesssim \frac{1}{32} \mathcal{M}_{\mathrm{S}}^{2} .
\end{gathered}
$$

These bounds on $\tau, \sigma$ do not have the status of exact analytical results (hence the symbol $\lesssim$ ), but they are reliable representations of the numerical data [24]. Note that the same violation of the Svetlichny inequality gives a tighter lower bound on $\tau, \sigma$ than the Mermin inequality gives due to the fact that 2-1 separable states are eliminated. 


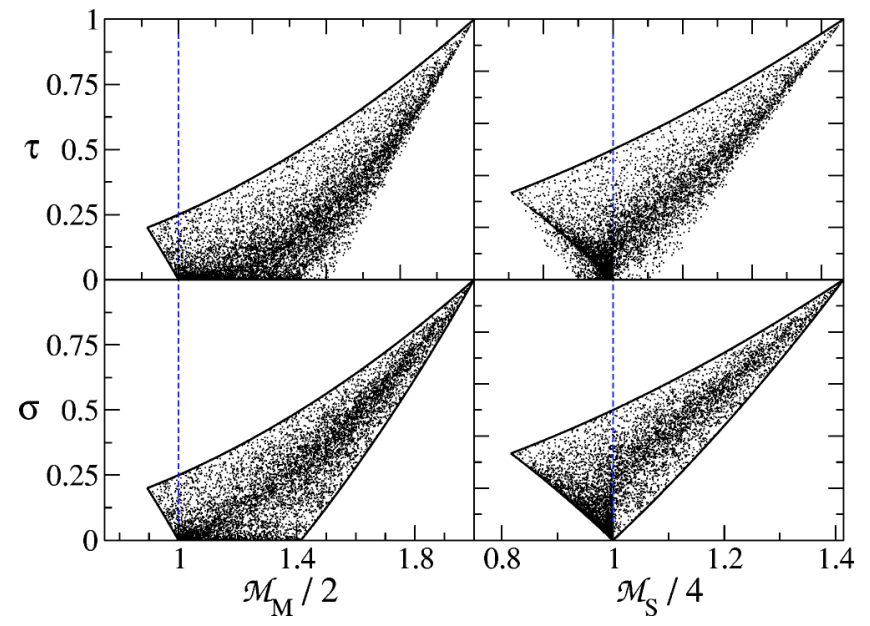

FIG. 2. Same as Fig. 1, but now for the general five-parameter state (4).

For the three-parameter states (14) in the GHZ class there is no advantage in using $\sigma$ over $\tau$. Both entanglement measures are bound in the same way by the Bell inequalities. That changes when we turn to the general five-parameter states (4), which also contain states in the W class. We see from Fig. 2 that the bounds (15) and (16) still apply to $\sigma$. However, the tangle $\tau$ drops below the previous lower bound due to the fact that it cannot distinguish W states from separable states.

In conclusion, we have constructed an entanglement monotone $\sigma$ for three qubits which, unlike the tangle $\tau$, can detect entanglement of both the GHZ and W types. We have investigated numerically the relation between the entanglement measures $\sigma, \tau$ and the maximal violation of Bell inequalities (both of the Mermin and Svetlichny form). The upper and lower bounds reported here have already been put to use in the design of a protocol for the detection of tripartite entanglement in the Fermi sea [23]. Alternatively, if one wants to do better than a bound, one could use the interferometric circuit proposed recently for the tangle [25], which, with a small modification, can be used to measure $\sigma$ as well.

We thank W. K. Wootters for drawing our attention to the merits of the Svetlichny inequality. This work was supported by the Dutch Science Foundation NWO/FOM and by the U.S. Army Research Office (Grant No. DAAD 19-02-10086).
[1] J. S. Bell, Physics (Long Island City, N.Y.) 1, 195 (1964).

[2] J. F. Clauser, M. A. Horne, A. Shimony, and R. A. Holt, Phys. Rev. Lett. 23, 880 (1969).

[3] N. Gisin, Phys. Lett. A 154, 201 (1991).

[4] A. Aspect, P. Grangier, and G. Roger, Phys. Rev. Lett. 47, 460 (1981).

[5] W. K. Wootters, Phys. Rev. Lett. 80, 2245 (1998).

[6] V. Coffman, J. Kundu, and W. K. Wootters, Phys. Rev. A 61, 052306 (2000).

[7] D. M. Greenberger, M. Horne, and A. Zeilinger, in Bell's Theorem, Quantum Theory and Conceptions of the Universe, edited by M. Kafatos (Kluwer, Dordrecht, 1989).

[8] N. D. Mermin, Phys. Rev. Lett. 65, 1838 (1990).

[9] V. Scarani and N. Gisin, J. Phys. A 34, 6043 (2001).

[10] G. Svetlichny, Phys. Rev. D 35, 3066 (1987).

[11] P. Mitchell, S. Popescu, and D. Roberts, quant-ph/0202009.

[12] D. Collins, N. Gisin, S. Popescu, D. Roberts, and V. Scarani, Phys. Rev. Lett. 88, 170405 (2002).

[13] M. Seevinck and G. Svetlichny, Phys. Rev. Lett. 89, 060401 (2002).

[14] W. Dür, G. Vidal, and J. I. Cirac, Phys. Rev. A 62, 062314 (2000).

[15] D. A. Meyer and N. R. Wallach, J. Math. Phys. 43, 4273 (2002). Their entanglement measure $Q$ can be expressed in terms of concurrences by $Q=\frac{1}{3}\left(\mathcal{C}_{A(B C)}^{2}+\mathcal{C}_{B(A C)}^{2}+\mathcal{C}_{C(A B)}^{2}\right)$. We have not found a simple relation between $Q$ and the Bell inequalities. However, the entanglement monotone $\mu=(\tau$
$+Q) / 2$ does satisfy the simple bounds $\left|\mathcal{M}_{\mathrm{M}}^{2} / 12-1 / 3\right| \lesssim \mu$ $\lesssim \mathcal{M}_{\mathrm{M}}^{2} / 16$.

[16] A. Acín, A. Andrianov, L. Costa, E. Jané, J. I. Latorre, and R. Tarrach, Phys. Rev. Lett. 85, 1560 (2000); A. Acín, A. Andrianov, E. Jané, and R. Tarrach, J. Phys. A 34, 6725 (2001).

[17] R. M. Gingrich, Phys. Rev. A 65, 052302 (2002).

[18] A. Sudbery, J. Phys. A 34, 643 (2001).

[19] We have also studied an alternative entanglement measure $\sigma^{\prime}=\min \left(\mathcal{C}_{X(Y Z)}^{2}-\mathcal{C}_{X Y}^{2}\right)=\tau+\min C_{X Y}^{2}$. Both $\sigma$ and $\sigma^{\prime}$ are capable of distinguishing $\mathrm{W}$ states from separable states, and they are bound in the same way by the Bell inequalities. We focus on $\sigma$ rather than $\sigma^{\prime}$ because we have no proof that $\sigma^{\prime}$ is an entanglement monotone.

[20] M. Żukowski, Č. Brukner, W. Laskowski, and M. Wieśniak, Phys. Rev. Lett. 88, 210402 (2002).

[21] J.-L. Chen, C.-F. Wu, L. C. Kwek, and C. H. Oh, quant-ph/ 0311180.

[22] A. Zeilinger, M. A. Horne, H. Weinfurter, and M. Żukowski, Phys. Rev. Lett. 78, 3031 (1997); J.-W. Pan, D. Bouwmeester, M. Daniell, H. Weinfurter, and A. Zeilinger, Nature (London) 403, 515 (2000).

[23] C. W. J. Beenakker, C. Emary, and M. Kindermann, Phys. Rev. B (to be published).

[24] The upper bounds on $\tau$ in Eqs. (15) and (16) are exact lower bounds on $\mathcal{M}_{\mathrm{M}}$ and $\mathcal{M}_{\mathrm{S}}$, as may be seen by evaluating $\mathcal{E}$ and $\mathcal{E}^{\prime}$ with $\boldsymbol{a}=\boldsymbol{b}=\boldsymbol{c}=(0,1,0)$, and $\boldsymbol{a}^{\prime}=\boldsymbol{b}^{\prime}=\boldsymbol{c}^{\prime}=(1,0,0)$.

[25] H. A. Carteret, quant-ph/0309212. 